\title{
Soliton diffusion on the classical, isotropic Heisenberg chain
}

\author{
M. Meister ${ }^{1, a}$, F.G. Mertens ${ }^{1}$, and A. Sánchez ${ }^{2}$ \\ 1 Physikalisches Institut, Universität Bayreuth, 95440 Bayreuth, Germany \\ 2 Grupo Interdisciplinar de Sistemas Complicados (GISC), Departamento de Matemáticas, Universidad Carlos III de Madrid, \\ Edificio Sabatini, Avenida de la Universidad 30, 28911 Leganés, Madrid, Spain
}

Received 23 November 2000 and Received in final form 31 January 2001

\begin{abstract}
We investigate the diffusive motion of a solitary wave on a classical, isotropic, ferromagnetic Heisenberg spin chain with nearest-neighbour exchange interaction. The spins are coupled magnetically to Gaussian white noise and are subject to Gilbert damping. The noise induces a collective, stochastic time evolution of the solitary wave. Within a continuum version of the model we employ implicit collective variables to describe this stochastic behaviour. Thermally excited magnons are disregarded. We derive stochastic equations of motion for the collective variables and solve them numerically, in particular to obtain their variances as functions of time. These results are compared to data from spin dynamics simulations of a discrete chain. For some of the collective variables we find good agreement with respect to the long time behaviour, whereas for other variables the agreement is only qualitative; reasons for this are given. For shorter times we derive analytical expressions for the variances of the collective variables, which also agree well with spin dynamics.
\end{abstract}

PACS. 05.40.-a Fluctuation phenomena, random processes, noise, and Brownian motion - 75.10.Hk Classical spin models -05.45. Yv Solitons

\section{Introduction}

Throughout physics the importance of coherent excitations in nonlinear systems - solitons and solitary waves are the most widely known - is well recognized. As it is impossible to list but the most important publications in this field, we only give a few sample references. An introductory discussion of solitary excitations and a summarizing overview of typical problems can be found in [1]. In [2], an exhaustive review of solitons in magnetic systems is given. More recent publications include [3-8]. A stronger emphasis on particle physics is put in [9].

Amongst all coherent excitations, solitons are very special. These excitations of localized energy density arise as solutions of integrable wave equations. Integrable physical systems usually are strong idealizations, because in most more realistic systems perturbations which spoil integrability will be present. There are many cases however, in which the perturbed, non-integrable system still exhibits coherent excitations of localized energy density, now no longer solitons but solitary waves. The soliton of the unperturbed system can often serve as a good approximation to these solitary waves and as a starting point for the treatment of the perturbed system; see [10-13] for example. Of course one can also start with a non-integrable system that exhibits solitary waves and study the effects

\footnotetext{
a e-mail: Matthias.Meister@uni-bayreuth.de
}

of perturbations there. The most prominent example of this type is the $\phi^{4}$ system $[3,4,14-16]$.

Due to their omnipresence, finite temperatures are a particularly important type of perturbation. The effects of temperature are often modelled by (white) noise coupled to the system as a stochastic perturbation. In these systems, the diffusion of the solitary excitations is one of the problems investigated; publications on this issue include [17-19] and references therein.

The dynamics of coherent excitations is often investigated in terms of collective variables, a technique which takes advantage of the particle-like nature of the excitations. If extended modes (linear waves, phonons, magnons) are neglected, as will be done in this work, this technique affords a reduction of the large number of degrees of freedom to a small number of collective variables describing the coherent excitation [20,21]. Moreover, investigations were carried out $[22,23]$ in which the total number of degrees of freedom was not reduced, but where collective variables appeared as a subset of a set of variables which had been specially tailored for describing a system in the presence of a particle-like excitation.

When using collective variables to study coherent excitations, there is, in general, the choice [24] between explicit collective variables, which are defined directly, i.e. explicitly, as functions of the degrees of freedom of the system, and implicit collective variables, which appear as variables in shape-functions describing the configuration 
of the system. Explicit variables have the advantage that they are clearly defined in terms of the degrees of freedom of the system; the definitions can be used unambiguously in calculations as well as in simulations. However, the structure of solitons and solitary excitations is usually parametrized by implicit collective variables. These variables appear within a function describing the excitation in question and have to be isolated from this function in one way or another before their dynamics can be investigated; it is not always possible to reexpress these variables in an explicit way.

In this article implicit collective variables are introduced to study the stochastic dynamics of solitary excitations on the classical Heisenberg spin chain with ferromagnetic, isotropic, nearest neighbour exchange interaction; in [25] an explicit collective variable, the position $X$ of the excitation, was used towards the same end. We will employ both a discrete version of the model and a continuum one and will alternate freely between these two versions throughout the paper. Spin dynamics simulations will be carried out for the discrete model, while analytical calculations will be done in the continuum chain. We restrict our investigations to solitary waves of a width considerably larger than the lattice constant (set equal to 1 ) of the discrete model, so a continuum approximation for analytical calculations of solitary wave dynamics is justified. As we neglect magnons altogether, we need not worry about differences in magnon dispersion relations between the discrete and the continuum case. Our aim is to derive stochastic equations of motion for the collective variables describing a solitary excitation, to use these equations for predicting the variances of the collective variables and to check the predictions by spin dynamics simulations.

The Hamiltonian of the unperturbed model in the discrete case is

$$
H=-J \sum_{n=1}^{N-1} \boldsymbol{S}_{n} \cdot \boldsymbol{S}_{n+1} \quad(J>0),
$$

which in the continuum version becomes

$$
H=\frac{J}{2} \int \partial_{r} \boldsymbol{S} \cdot \partial_{r} \boldsymbol{S} \mathrm{d} r
$$

where $r$ denotes the coordinate along the chain. We are using dimensionless quantities: $J$ gives the exchange coupling in units of a positive scale $J_{0}$ and $S$ measures the spinlength in units of a scale $S_{0}$. As a result, $J_{0} S_{0}^{2}$ is the unit of energy and $\hbar /\left(J_{0} S_{0}^{2}\right)$ the unit of time. In (2) a constant ground state energy has been subtracted, so this expression gives the excitation energy above the ground state. Furthermore, we have moved from a discrete chain of $N$ spins to a continuum one of infinite length. We assume that the spin field in the continuum case tends towards the same ground state configuration (there is an infinite degeneracy for the case of an isotropic chain) as we approach infinity, on either side of the excitation. For the discrete chain we use free boundary conditions.

The perturbed spin dynamics of the chain is governed by the Landau-Lifshitz equation with Gilbert damping of strength $\varepsilon$ and magnetically coupled white noise $\boldsymbol{b}$ :

$$
\frac{\mathrm{d}}{\mathrm{d} t} \boldsymbol{S}+\varepsilon \boldsymbol{S} \times \frac{\mathrm{d}}{\mathrm{d} t} \boldsymbol{S}=-\boldsymbol{S} \times \boldsymbol{B}-\boldsymbol{S} \times \boldsymbol{b} .
$$

This equation has to be considered in Stratonovich interpretation $[26,27]$; the question of the correct interpretation is important here because the magnetic coupling leads to multiplicative couplings between the components of the spins and of the noise. For the continuum model, $\boldsymbol{S}$ denotes the spin field; in the discrete case, (3) gives the equation of motion for each individual spin of the chain. The quantity $\boldsymbol{B}$ is the effective magnetic field, arising from the interaction amongst the spins. It is given by

$$
B_{n}^{j}=\frac{\partial H}{\partial S_{n}^{j}} \quad \text { and } \quad \boldsymbol{B}=-J \partial_{r}^{2} \boldsymbol{S}-2 J \boldsymbol{S},
$$

respectively; $B_{n}^{j}$ denotes the component $j$ at the site $n$. The noise $\boldsymbol{b}$ is Gaussian and white, with properties

$$
\begin{aligned}
& \left\langle b^{i}(r, t)\right\rangle=0 \\
& \left\langle b^{i}\left(r_{1}, t_{1}\right) b^{j}\left(r_{2}, t_{2}\right)\right\rangle=\sigma^{2} \delta^{i j} \delta\left(r_{1}-r_{2}\right) \delta\left(t_{1}-t_{2}\right) .
\end{aligned}
$$

Analogous relations obtain in the discrete case. The perturbations are regarded as the effects of coupling the spins to a heat bath of temperature $T$. This requires $\sigma^{2}=2 \varepsilon k_{\mathrm{B}} T, k_{\mathrm{B}}$ being Boltzmann's constant. A more detailed discussion of these perturbations can be found in $[25,26]$. For the unperturbed, isotropic, continuum Heisenberg chain (3) reduces to

$$
\partial_{t} \boldsymbol{S}=J \boldsymbol{S} \times \partial_{r}^{2} \boldsymbol{S} .
$$

This equation has solitons amongst its solutions [28-31], which in the representation

$$
S=S\left(\sqrt{1-\Psi^{2}} \cos \Phi, \sqrt{1-\Psi^{2}} \sin \Phi, \Psi\right)
$$

for the Cartesian spin components can be written as

$$
\begin{aligned}
\Psi(r, t)= & 1-A\left[\operatorname{sech}\left(\frac{r-X}{\Gamma}\right)\right]^{2}, \\
\Phi(r, t)= & \Phi_{0}+\sqrt{\frac{2}{A}-1 \frac{r-X}{\Gamma}} \\
& +\arctan \left[\sqrt{\frac{A}{2-A}} \tanh \left(\frac{r-X}{\Gamma}\right)\right],
\end{aligned}
$$

where $\Phi_{0}=\phi_{0}+J S \frac{2}{A \Gamma^{2}} t$ and $X=X_{0}+J S \frac{2}{\Gamma} \sqrt{\frac{2}{A}-1} t$. $A$ is the amplitude, $\Gamma$ is the width, and $X$ is the position of the soliton. The excitation energy of the soliton, as calculated from (2), is $4 J S^{2} / \Gamma$.

In Section 2 we neglect damping and derive stochastic equations of motion for the collective variables $X, A$ and $\Gamma$. We perturbatively find an analytic expression for the time dependence of the variances of these variables. Gilbert damping is taken into account in Section 3. Stochastic equations of motion for collective variables describing general coherent excitations in $d$-dimensional spin 
systems are found, and then specialized to the solitary excitations given by (8). This yields equations of motion for the collective variables $X, \Phi_{0}, A$ and $\Gamma$. These equations are not solved analytically, but numerical solutions are presented in Section 4. There we also compare the results obtained in this way with spin dynamics simulations and with the results derived in Section 2. The short time behaviour of our spin dynamics data turns out to be well described by the analytical results of Section 2, while the numerical solutions of the equations obtained in Section 3 show a different behaviour. For long times, the latter results agree very well with spin dynamics in the case of the variables $X$ and $\Phi_{0}$, but for $A$ and $\Gamma$ the agreement is not satisfactory. These findings are discussed. Finally, Section 5 gives our conclusions.

\section{Stochastic equations of motion without damping}

In this section we calculate the variance of the collective variables $X, A$ and $\Gamma$. The approach chosen here corresponds to the way the position $X$ is determined from spin dynamics simulations (see the appendices). We do not take into account the damping term for reasons that will become evident below. This restricts the results of this approach to time scales for which the effects of damping can be neglected. Thus, equation (3) takes the form

$$
\frac{\mathrm{d}}{\mathrm{d} t} \boldsymbol{S}=J \boldsymbol{S} \times \partial_{r}^{2} \boldsymbol{S}-\boldsymbol{S} \times \boldsymbol{b} .
$$

Here we use only the $z$-component of this equation and an ansatz for the spin field $\boldsymbol{S}$ which is given by (8), with time dependent collective variables $X, A, \Gamma$ and $\Phi_{0}$. Noting that $S^{z}=S \Psi$ is independent of $\Phi_{0}$, we find

$$
\frac{\partial S^{z}}{\partial X} \dot{X}+\frac{\partial S^{z}}{\partial A} \dot{A}+\frac{\partial S^{z}}{\partial \Gamma} \dot{\Gamma}=J\left[\boldsymbol{S} \times \partial_{r}^{2} \boldsymbol{S}\right]^{z}-[\boldsymbol{S} \times \boldsymbol{b}]^{z} .
$$

A Gilbert damping term would produce an additional term proportional to $\dot{\Phi}_{0}$ on the left, which would subsequently cause problems, see below. Now we multiply this equation with $\frac{\partial S^{z}}{\partial X}, \frac{\partial S^{z}}{\partial A}$ and $\frac{\partial S^{z}}{\partial \Gamma}$, respectively, and integrate over the chain, obtaining the system of equations

$$
\begin{aligned}
S^{2}\left[\begin{array}{ccc}
\frac{16}{15} \frac{A^{2}}{\Gamma} & 0 & 0 \\
0 & \frac{4}{3} \Gamma & \frac{2}{3} A \\
0 & \frac{2}{3} A \frac{4 \pi^{2}}{45} \frac{A^{2}}{\Gamma}
\end{array}\right]\left[\begin{array}{c}
\dot{X} \\
\dot{A} \\
\dot{\Gamma}
\end{array}\right] & =\left[\begin{array}{c}
J S^{3} \frac{32}{15} \frac{A^{\frac{3}{2}} \sqrt{2-A}}{\Gamma^{2}} \\
0 \\
0
\end{array}\right] \\
& -\left[\begin{array}{c}
\int \frac{\partial S^{z}}{\partial X}(\boldsymbol{S} \times \boldsymbol{b})^{z} \mathrm{~d} r \\
\int \frac{\partial S^{z}}{\partial A}(\boldsymbol{S} \times \boldsymbol{b})^{z} \mathrm{~d} r \\
\int \frac{\partial S^{z}}{\partial \Gamma}(\boldsymbol{S} \times \boldsymbol{b})^{z} \mathrm{~d} r
\end{array}\right] .
\end{aligned}
$$

As $S^{z}$ does not depend on $\Phi_{0}$, we cannot multiply by the corresponding derivative. Had we taken into account the damping term in (3), we would have three linear equations for four unknowns, namely $\dot{X}, \dot{\Phi}_{0}, \dot{A}$ and $\dot{\Gamma}$.

After inversion of system (11) we obtain:

$$
\begin{aligned}
\dot{X} & =\frac{2 J S}{\Gamma} \sqrt{\frac{2}{A}-1}+\eta_{X}, \\
\dot{A} & =\eta_{A}, \\
\dot{\Gamma} & =\eta_{\Gamma} .
\end{aligned}
$$

Here the quantities $\eta$ are effective stochastic forces, which, on the level of collective variables, reflect the effects of the noise coupled to the spins. Assuming that the collective variables are stochastically independent of the noise $\boldsymbol{b}$ (we postpone a detailed discussion of this assumption to Sect. 3), the $\eta$ can be found to satisfy $\left\langle\eta_{U}\right\rangle=0$ for $U=X, A, \Gamma$ and

$$
\left\langle\eta_{X}\left(t_{1}\right) \eta_{X}\left(t_{2}\right)\right\rangle=\sigma^{2} \delta\left(t_{1}-t_{2}\right) \frac{5}{14}\left\langle\Gamma \frac{3-A}{A}\right\rangle,
$$

$$
\begin{gathered}
\left\langle\eta_{A}\left(t_{1}\right) \eta_{A}\left(t_{2}\right)\right\rangle=\sigma^{2} \delta\left(t_{1}-t_{2}\right) \frac{1}{\left(4 \pi^{2}-15\right)^{2}}\left\langle\frac { A } { \Gamma } \left\{\frac{96}{5} \pi^{4}\right.\right. \\
\left.\left.-\frac{312}{7} \pi^{2}-180+\frac{A}{7}\left[-\frac{288}{5} \pi^{4}+96 \pi^{2}+620\right]\right\}\right\rangle
\end{gathered}
$$

$$
\begin{aligned}
&\left\langle\eta_{\Gamma}\left(t_{1}\right) \eta_{\Gamma}\left(t_{2}\right)\right\rangle=\sigma^{2} \delta\left(t_{1}-t_{2}\right) \frac{8}{15}\left(\frac{45}{4 \pi^{2}-15}\right)^{2} \\
& \times\left\langle\frac{\Gamma}{A}\left\{-1+\frac{4 \pi^{2}}{21}-\frac{A}{63}\left(4 \pi^{2}-\frac{62}{3}\right)\right\}\right\rangle
\end{aligned}
$$

$$
\begin{aligned}
& \left\langle\eta_{A}\left(t_{1}\right) \eta_{\Gamma}\left(t_{2}\right)\right\rangle=\sigma^{2} \delta\left(t_{1}-t_{2}\right) \frac{9}{\left(4 \pi^{2}-15\right)^{2}} \\
& \times\left\{80-\frac{352}{21} \pi^{2}+\frac{\langle A\rangle}{21}\left(152 \pi^{2}-\frac{2050}{3}\right)\right\} \\
& \left\langle\eta_{X}\left(t_{1}\right) \eta_{A}\left(t_{2}\right)\right\rangle=0, \quad\left\langle\eta_{X}\left(t_{1}\right) \eta_{\Gamma}\left(t_{2}\right)\right\rangle=0 .
\end{aligned}
$$

In Section 3 we will again calculate correlations of stochastic forces, and there we will give a more detailed derivation, which is parallel to the steps not shown here. Introducing quantities $\Sigma_{U V}$ by

$$
\left\langle\eta_{U}\left(t_{1}\right) \eta_{V}\left(t_{2}\right)\right\rangle=\sigma^{2} \delta\left(t_{1}-t_{2}\right)\left\langle\Sigma_{U V}(A, \Gamma)\right\rangle
$$

and mutually independent Wiener processes $W_{X}, W_{1}$ and $W_{2}$, we can express the equations of motion (12) as follows:

$$
\begin{aligned}
\mathrm{d} X & =\frac{2 J S}{\Gamma} \sqrt{\frac{2}{A}-1} \mathrm{~d} t+\sigma \alpha(A, \Gamma) \mathrm{d} W_{X}, \\
\mathrm{~d} A & =\sigma \beta(A, \Gamma) \mathrm{d} W_{1}, \\
\mathrm{~d} \Gamma & =\sigma \gamma_{1}(A, \Gamma) \mathrm{d} W_{1}+\sigma \gamma_{2}(A, \Gamma) \mathrm{d} W_{2},
\end{aligned}
$$


where $\alpha^{2}=\Sigma_{X X}, \beta^{2}=\Sigma_{A A}, \gamma_{1} \beta=\Sigma_{A \Gamma}$ and $\gamma_{1}^{2}+\gamma_{2}^{2}=$ $\Sigma_{\Gamma \Gamma}$. The set of equations (19) is problematic, however: The stochastic quantities $A$ and $\Gamma$ may assume unphysical values, i.e. the amplitude $A$ may grow beyond its maximum 2 or assume negative values, and likewise the width can become negative. In any event, the probability for this to occur is very small, if we consider only weak noise (low temperatures) and short times. Here it is important to recall that the set (19) has been derived from an ansatz describing a solitary wave, where the effects of the perturbations have been taken into account only by allowing for a time dependence of the collective variables; all extended excitations (magnons) have been neglected. Furthermore, we have neglected some correlations in the derivation of the set (19) by assuming that the noise is stochastically independent of the collective variables. These assumptions appear justified as long as the noise is weak.

Since the derivation of (19) included approximations anyway (weak noise and short times), we do not solve the equations (19) exactly. Instead, we resort to a perturbative technique. A small noise expansion [27] $X=X_{0}+\sigma X_{1}+$ $\sigma^{2} X_{2}+\ldots$, and similarly for $A$ and $\Gamma$, yields for the first nontrivial order $\left(\sigma^{1}\right)$ :

$$
\begin{aligned}
\mathrm{d} X_{1}= & -\frac{2 J S}{\Gamma_{0}^{2}} \sqrt{\frac{2}{A_{0}}-1} \Gamma_{1} \mathrm{~d} t \\
& -\frac{2 J S}{A_{0}^{2} \Gamma_{0}}\left(\frac{2}{A_{0}}-1\right)^{-\frac{1}{2}} A_{1} \mathrm{~d} t+\alpha\left(A_{0}, \Gamma_{0}\right) \mathrm{d} W_{X} \\
\mathrm{~d} A_{1}= & \beta\left(A_{0}, \Gamma_{0}\right) \mathrm{d} W_{1} \\
\mathrm{~d} \Gamma_{1}= & \gamma_{1}\left(A_{0}, \Gamma_{0}\right) \mathrm{d} W_{1}+\gamma_{2}\left(A_{0}, \Gamma_{0}\right) \mathrm{d} W_{2}
\end{aligned}
$$

From this we find

$$
\begin{gathered}
A_{1}(t)=\beta\left(A_{0}, \Gamma_{0}\right)\left[W_{1}(t)-W_{1}(0)\right], \\
\Gamma_{1}(t)=\gamma_{1}\left(A_{0}, \Gamma_{0}\right)\left[W_{1}(t)-W_{1}(0)\right] \\
+\gamma_{2}\left(A_{0}, \Gamma_{0}\right)\left[W_{2}(t)-W_{2}(0)\right],
\end{gathered}
$$

and

$$
\begin{gathered}
X_{1}(t)=-\frac{2 J S}{\Gamma_{0}^{2}} \sqrt{\frac{2}{A_{0}}-1} \int_{0}^{t}\left\{\gamma _ { 1 } ( A _ { 0 } , \Gamma _ { 0 } ) \left[W_{1}\left(t^{\prime}\right)\right.\right. \\
\left.\left.-W_{1}(0)\right]+\gamma_{2}\left(A_{0}, \Gamma_{0}\right)\left[W_{2}\left(t^{\prime}\right)-W_{2}(0)\right]\right\} \mathrm{d} t^{\prime} \\
-\frac{2 J S}{A_{0}^{2} \Gamma_{0}}\left(\frac{2}{A_{0}}-1\right)^{-\frac{1}{2}} \int_{0}^{t} \beta\left(A_{0}, \Gamma_{0}\right)\left[W_{1}\left(t^{\prime}\right)-W_{1}(0)\right] \mathrm{d} t^{\prime} \\
+\alpha\left(A_{0}, \Gamma_{0}\right)\left[W_{X}(t)-W_{X}(0)\right]
\end{gathered}
$$

where $A_{0}$ and $\Gamma_{0}$ denote the constant initial values. To this order we have $\operatorname{Var}[X(t)]=\sigma^{2}\left\langle X_{1}(t)^{2}\right\rangle$ and thus we finally obtain, with $\Sigma_{U V}$ evaluated at $U_{0}, V_{0}$ :

$$
\begin{aligned}
\operatorname{Var}[X(t)]= & \sigma^{2} \Sigma_{X X} t+4 J^{2} S^{2} \frac{\sigma^{2}}{3 \Gamma_{0}^{2}}\left\{\frac{1}{\Gamma_{0}^{2}}\left(\frac{2}{A_{0}}-1\right) \Sigma_{\Gamma \Gamma}\right. \\
& \left.+\frac{\Sigma_{A A}}{A_{0}^{4}\left(\frac{2}{A_{0}}-1\right)}+\frac{2 \Sigma_{A \Gamma}}{A_{0}^{2} \Gamma_{0}}\right\} t^{3} .
\end{aligned}
$$

The term linear in $t$ derives from the direct effect of the noise on the position $X$, expressed by the stochastic force $\eta_{X}$. The cubic term is the result of an indirect effect: The noise also changes $A$ and $\Gamma$ stochastically, but these two variables determine the velocity of the excitation. The position, in turn, is, up to a constant, given by the time integral of the velocity; therefore differences in the velocity (e.g. those induced by the noise) will lead to differences in the position that grow with time. Thus the time dependence of the variance of the position can be expected to include higher than linear powers of time. Within the first order approximation applied above, the velocity is described by a Wiener process, so the position contains a time integral of a Wiener process. The cubic term in (24) straightforwardly derives from this integral. Similar superdiffusive behaviour was found in [32]. There the effects of noise on the position of a vortex in a two-dimensional easy-plane Heisenberg model were studied; the indirect effect of the noise on the position originated from an external force acting on the vortex. The force was such that it enlarged differences between trajectories. Consequently, some of the variances of interest in that case were found to grow faster than linear in time.

For $A$ and $\Gamma$ we obtain

$$
\begin{aligned}
\operatorname{Var}[A(t)] & =\sigma^{2} \Sigma_{A A}\left(A_{0}, \Gamma_{0}\right) t, \\
\operatorname{Var}[\Gamma(t)] & =\sigma^{2} \Sigma_{\Gamma \Gamma}\left(A_{0}, \Gamma_{0}\right) t .
\end{aligned}
$$

As we will see in Section 4, the predictions for $A$ and $\Gamma$ agree with the spin dynamics results only for a short time $(t \lesssim 100)$, whereas the prediction for the position is good up to several hundreds of time units. Results describing the long time behaviour will be obtained in the following section.

\section{Stochastic equations of motion with damping}

In this section we derive equations of motion that account for the Gilbert damping term in (3) and hence can be expected to apply to longer times than the results of Section 2. As a first step, we derive general equations of motion for collective variables describing coherent excitations in a $d$-dimensional spin system, and afterwards we specialize to the Heisenberg chain. We set out from the ansatz

$$
\boldsymbol{S}(r, t)=\boldsymbol{S}_{\mathrm{c}}\left(r, \ldots, U_{i}(t), \ldots\right),
$$

where $r$ denotes a point in $d$-dimensional space, and $U_{i}$ is one of finitely, say $N$, many collective coordinates. The function $\boldsymbol{S}_{\mathrm{c}}$ describes a particular type of coherent excitation in terms of $r$ and the $U_{1}, \ldots, U_{N}$. From (26) we find immediately

$$
\frac{\partial}{\partial t} \boldsymbol{S}=\sum_{i=1}^{N} \frac{\partial \boldsymbol{S}_{\mathrm{c}}}{\partial U_{i}} \dot{U}_{i}
$$


and using this in the perturbed Landau-Lifshitz equation (3), we arrive at

$$
\sum_{i=1}^{N}\left(\frac{\partial \boldsymbol{S}_{\mathrm{c}}}{\partial U_{i}}+\varepsilon \boldsymbol{S}_{\mathrm{c}} \times \frac{\partial \boldsymbol{S}_{\mathrm{c}}}{\partial U_{i}}\right) \dot{U}_{i}=-\boldsymbol{S}_{\mathrm{c}} \times(\boldsymbol{B}+\boldsymbol{b}) .
$$

The next step is to isolate from these equations the time derivatives of the $U_{i}$. In analogy to [33], along the lines of $[26,32]$, we first take the vector product of (28) with $\frac{\partial \boldsymbol{S}_{\mathrm{c}}}{\partial U_{k}}$, then form the inner product of the result with $\boldsymbol{S}_{\mathrm{c}}$ and integrate over all space, obtaining

$$
\begin{aligned}
& \sum_{i=1}^{N} \int \boldsymbol{S}_{\mathrm{c}} \cdot\left[\frac{\partial \boldsymbol{S}_{\mathrm{c}}}{\partial U_{k}} \times \frac{\partial \boldsymbol{S}_{\mathrm{c}}}{\partial U_{i}}+\varepsilon \frac{\partial \boldsymbol{S}_{\mathrm{c}}}{\partial U_{k}} \times\left(\boldsymbol{S}_{\mathrm{c}} \times \frac{\partial \boldsymbol{S}_{\mathrm{c}}}{\partial U_{i}}\right)\right] \mathrm{d}^{d} r \dot{U}_{i} \\
&=-\int \boldsymbol{S}_{\mathrm{c}} \cdot\left[\frac{\partial \boldsymbol{S}_{\mathrm{c}}}{\partial U_{k}} \times\left(\boldsymbol{S}_{\mathrm{c}} \times(\boldsymbol{B}+\boldsymbol{b})\right)\right] \mathrm{d}^{d} r
\end{aligned}
$$

for each of the collective variables $U_{k}$. We introduce a vector $\boldsymbol{C}:=\boldsymbol{C}^{\text {in }}+\boldsymbol{C}^{\mathrm{ex}}$ with

$$
\begin{aligned}
C_{k}^{\mathrm{in}} & :=-\int \boldsymbol{S}_{\mathrm{c}} \cdot\left[\frac{\partial \boldsymbol{S}_{\mathrm{c}}}{\partial U_{k}} \times\left(\boldsymbol{S}_{\mathrm{c}} \times \boldsymbol{B}\right)\right] \mathrm{d}^{d} r, \\
C_{k}^{\mathrm{ex}} & :=-\int \boldsymbol{S}_{\mathrm{c}} \cdot\left[\frac{\partial \boldsymbol{S}_{\mathrm{c}}}{\partial U_{k}} \times\left(\boldsymbol{S}_{\mathrm{c}} \times \boldsymbol{b}\right)\right] \mathrm{d}^{d} r
\end{aligned}
$$

where $C^{\text {in }}$ is the intrinsic part, containing the effective magnetic field $\boldsymbol{B}$ that arises from the interaction amongst the spins, and $C^{\text {ex }}$ is the external part, proportional to the external noise $\boldsymbol{b}$. As $\boldsymbol{S}_{\mathrm{c}} \cdot\left(\frac{\partial \boldsymbol{S}_{\mathrm{c}}}{\partial U_{k}} \times \frac{\partial \boldsymbol{S}_{\mathrm{c}}}{\partial U_{i}}\right)=-\frac{\partial \boldsymbol{S}_{\mathrm{c}}}{\partial U_{k}} \cdot\left(\boldsymbol{S}_{\mathrm{c}} \times \frac{\partial \boldsymbol{S}_{\mathrm{c}}}{\partial U_{i}}\right)$ and $\frac{\partial \boldsymbol{S}_{\mathrm{c}}}{\partial U_{k}} \times\left(\boldsymbol{S}_{\mathrm{c}} \times \frac{\partial \boldsymbol{S}_{\mathrm{c}}}{\partial U_{i}}\right)=\boldsymbol{S}_{\mathrm{c}}\left(\frac{\partial \boldsymbol{S}_{\mathrm{c}}}{\partial U_{k}} \cdot \frac{\partial \boldsymbol{S}_{\mathrm{c}}}{\partial U_{i}}\right)$, because $\boldsymbol{S}_{\mathrm{c}} \cdot \frac{\partial \boldsymbol{S}_{\mathrm{c}}}{\partial U_{k}}=\frac{1}{2} \frac{\partial}{\partial U_{k}}\left(\boldsymbol{S}_{\mathrm{c}} \cdot \boldsymbol{S}_{\mathrm{c}}\right)$ vanishes due to the invariance of the spinlength $S$, (29) can be written as

$$
\sum_{i=1}^{N}\left[-M_{2, k i}+\varepsilon S^{2} M_{1, k i}\right] \dot{U}_{i}=C_{k}
$$

where

$$
\begin{aligned}
M_{1, k i} & :=\int \frac{\partial \boldsymbol{S}_{\mathrm{c}}}{\partial U_{k}} \cdot \frac{\partial \boldsymbol{S}_{\mathrm{c}}}{\partial U_{i}} \mathrm{~d}^{d} r, \text { and } \\
M_{2, k i} & :=\int \frac{\partial \boldsymbol{S}_{\mathrm{c}}}{\partial U_{k}} \cdot\left(\boldsymbol{S}_{\mathrm{c}} \times \frac{\partial \boldsymbol{S}_{\mathrm{c}}}{\partial U_{i}}\right) \mathrm{d}^{d} r .
\end{aligned}
$$

Introducing a matrix $L(\varepsilon)$ by $L(\varepsilon)_{k i}:=-M_{2, k i}+\varepsilon S^{2} M_{1, k i}$ we arrive at

$$
\dot{U}_{i}=\sum_{k=1}^{N}\left[L(\varepsilon)^{-1}\right]_{i k} C_{k}
$$

Defining $F_{i}:=\left[L(\varepsilon)^{-1} \boldsymbol{C}^{\text {in }}\right]_{i}$ and $f_{i}:=\left[L(\varepsilon)^{-1} \boldsymbol{C}^{\mathrm{ex}}\right]_{i}$ we finally obtain from (33):

$$
\dot{U}_{i}=F_{i}+f_{i} .
$$

On the level of collective coordinates the interaction of the constituents of the system is thus reflected by "intrinsic forces" $F_{i}$, whereas the quantities $f_{i}$ are effective stochastic forces, analogous to the $\eta$ s of Section 2. The correlations of the $f_{i}$ are given by (the $\varepsilon$-dependence of the matrix $L(\varepsilon)^{-1}$ is not written explicitly):

$$
\left\langle f_{i}\left(t_{1}\right) f_{j}\left(t_{2}\right)\right\rangle=\sum_{m=1}^{N} \sum_{k=1}^{N}\left\langle L_{i k}^{-1}\left(t_{1}\right) C_{k}^{\mathrm{ex}}\left(t_{1}\right) L_{j m}^{-1}\left(t_{2}\right) C_{m}^{\mathrm{ex}}\left(t_{2}\right)\right\rangle .
$$

Note that, by making the ansatz (26), we have already neglected magnons; however, the function $\boldsymbol{S}_{\mathrm{c}}$ depends on the collective variables, and these are stochastic quantities. Therefore the expectation values in (35) cannot be evaluated straightforwardly, because they involve products of functions of the collective variables and noise terms $\boldsymbol{b}$. As (3) is considered a Stratonovich equation, there could be correlations between the noise and the collective variables which may prevent an exact evaluation of (35).

On the other hand we can derive the Ito-version of (33). In this formulation, the noise terms are stochastically independent of the collective variables, but there will appear, in general, correction terms to $F_{i}$. These correction terms are of second order in the noise (i.e. $\propto \sigma^{2}$ ), whereas the $f_{i}$ are of first order in $\sigma$. For small values of $\sigma$ (weak noise) we can therefore neglect the correction terms in $F_{i}$ and treat the collective variables as stochastically independent of the noise. This allows us to split the expectation values of the products in (35) into a product of expectation values:

$$
\begin{aligned}
\left\langle f_{i}\left(t_{1}\right) f_{j}\left(t_{2}\right)\right\rangle=\sum_{k=1}^{N} \sum_{m=1}^{N}\left\langle L_{i k}^{-1}\left(t_{1}\right) L_{j m}^{-1}\left(t_{2}\right) S^{4}\right. \\
\left.\times \iint \sum_{h=1}^{3} \sum_{g=1}^{3} \frac{\partial S_{\mathrm{c}}^{h}}{\partial U_{k}}\left(t_{1}, r_{1}\right) \frac{\partial S_{\mathrm{c}}^{g}}{\partial U_{m}}\left(t_{2}, r_{2}\right)\right\rangle \\
\times\left\langle b^{h}\left(r_{1}, t_{1}\right) b^{g}\left(r_{2}, t_{2}\right)\right\rangle \mathrm{d}^{d} r_{1} \mathrm{~d}^{d} r_{2} .
\end{aligned}
$$

Using the properties of $\boldsymbol{b}$, equation (5), we find

$$
\begin{aligned}
\left\langle f_{i}\left(t_{1}\right) f_{j}\left(t_{2}\right)\right\rangle= & \sigma^{2} S^{4} \sum_{k=1}^{N} \sum_{m=1}^{N}\left\langle L_{i k}^{-1}\left(t_{1}\right) L_{j m}^{-1}\left(t_{1}\right)\right. \\
& \left.\times \int \frac{\partial \boldsymbol{S}_{\mathrm{c}}}{\partial U_{k}} \cdot \frac{\partial \boldsymbol{S}_{\mathrm{c}}}{\partial U_{m}}\left(t_{1}, r\right) d^{d} r\right\rangle \delta\left(t_{1}-t_{2}\right) \\
= & \sigma^{2} S^{4}\left\langle\left[L^{-1} M_{1}\left(L^{-1}\right)^{T}\right]_{i j}\right\rangle \delta\left(t_{1}-t_{2}\right) \\
= & :\left\langle D_{i j}\right\rangle \delta\left(t_{1}-t_{2}\right)
\end{aligned}
$$

where the superscript $T$ denotes the transpose. In a similar way it can be seen that $\left\langle f_{i}\right\rangle=0$. Thus, within the above approximations, the $f_{i}$ represent additive and white (i.e. the correlation time is zero) noise on the level of collective variables. Without the approximations, the expressions (35) could contain non-vanishing correlation time scales (i.e. the noise may be coloured). However, as the result (37) shows, these time scales become negligible for weak noise. Analogous statements apply to the results for the correlations of the $\eta \mathrm{s}$ in Section 2. 
In order to evaluate the components of the matrices $M$ and thence also of $L$ and its inverse, we use the representation (7) and obtain after some algebra

$$
\frac{\partial \boldsymbol{S}_{\mathrm{c}}}{\partial U_{k}} \cdot \frac{\partial \boldsymbol{S}_{\mathrm{c}}}{\partial U_{i}}=S^{2}\left[\frac{1}{1-\Psi_{\mathrm{c}}^{2}} \frac{\partial \Psi_{\mathrm{c}}}{\partial U_{k}} \frac{\partial \Psi_{\mathrm{c}}}{\partial U_{i}}+\left(1-\Psi_{\mathrm{c}}^{2}\right) \frac{\partial \Phi_{\mathrm{c}}}{\partial U_{k}} \frac{\partial \Phi_{\mathrm{c}}}{\partial U_{i}}\right]
$$

and

$$
\frac{\partial \boldsymbol{S}_{\mathrm{c}}}{\partial U_{k}} \cdot\left(\boldsymbol{S}_{\mathrm{c}} \times \frac{\partial \boldsymbol{S}_{\mathrm{c}}}{\partial U_{i}}\right)=S^{3}\left(\frac{\partial \Psi_{\mathrm{c}}}{\partial U_{k}} \frac{\partial \Phi_{\mathrm{c}}}{\partial U_{i}}-\frac{\partial \Psi_{\mathrm{c}}}{\partial U_{i}} \frac{\partial \Phi_{\mathrm{c}}}{\partial U_{k}}\right)
$$

Now we specialize to the isotropic, one-dimensional case and consider the soliton solutions (8) given above. For this type of excitation we use four collective variables, namely $X, \Phi_{0}, A$ and $\Gamma$. For an isotropic chain with nearest neighbour exchange, $\boldsymbol{B}$ is given by (4), so

$$
\begin{aligned}
C_{k}= & J \int\left[\frac{\partial}{\partial r}\left(\boldsymbol{S}_{\mathrm{c}} \times \frac{\partial}{\partial r} \boldsymbol{S}_{\mathrm{c}}\right)\right] \cdot\left(\boldsymbol{S}_{\mathrm{c}} \times \frac{\partial \boldsymbol{S}_{\mathrm{c}}}{\partial U_{k}}\right) \mathrm{d} r \\
& -S^{2} \int \boldsymbol{b} \cdot \frac{\partial \boldsymbol{S}_{\mathrm{c}}}{\partial U_{k}} \mathrm{~d} r
\end{aligned}
$$

Using the soliton solutions (8), the intrinsic parts can be evaluated further, eventually leading to the equations for the collective variables:

$$
\begin{gathered}
\dot{X}=\frac{2 J S}{\Gamma} \sqrt{\frac{2}{A}-1} \\
\times \frac{9\left[9+\left(\pi^{2}+6\right) \varepsilon^{2} S^{2}\right]}{\left[9\left(9+\varepsilon^{2} S^{2}\left(2 \pi^{2}+12\right)\right)\right]+\varepsilon^{4} S^{4} \pi^{2}\left(\pi^{2}+12\right)}+f_{1} \\
\dot{\Phi}_{0}=\frac{2 J S}{A \Gamma^{2}} \frac{9}{9+\varepsilon^{2} S^{2} \pi^{2}}+f_{2} \\
\dot{A}=\frac{36 J S^{2} \varepsilon}{\varepsilon^{2} S^{2}\left(\pi^{2}+12\right)+9} \frac{A-2}{\Gamma^{2}}+f_{3}
\end{gathered}
$$

$\dot{\Gamma}=-12 J S^{2} \varepsilon \frac{\left(\left(\pi^{2}-6\right) \varepsilon^{2} S^{2}+9\right) A-3\left(\pi^{2} \varepsilon^{2} S^{2}+9\right)}{\left(\pi^{2} \varepsilon^{2} S^{2}+9\right)\left(\left(\pi^{2}+12\right) \varepsilon^{2} S^{2}+9\right) \Gamma A}+f_{4}$.

For the correlations (37) of the stochastic forces it turns out that

$$
D=\left[\begin{array}{cccc}
D_{X X} & D_{X \Phi_{0}} & 0 & 0 \\
D_{X \Phi_{0}} & D_{\Phi_{0} \Phi_{0}} & 0 & 0 \\
0 & 0 & D_{A A} & D_{A \Gamma} \\
0 & 0 & D_{A \Gamma} & D_{\Gamma \Gamma}
\end{array}\right]
$$

Here the components depend on $\Gamma$ and $A$, which in turn depend on time:

$$
\begin{aligned}
D_{X X}= & \sigma^{2} \frac{\Gamma}{4 A} \\
& \times \frac{9 \pi^{2}(3-A)+54 A+\varepsilon^{2} S^{2} \pi^{2}\left(\pi^{2}+12\right)(3-A)}{\left(9+\varepsilon^{2} S^{2} \pi^{2}\right)\left[9+\varepsilon^{2} S^{2}\left(\pi^{2}+12\right)\right]}, \\
D_{X \Phi_{0}}= & \sigma^{2} \frac{3}{4} \sqrt{\frac{2}{A}-1} \frac{\pi^{2}}{A\left(9+\varepsilon^{2} S^{2} \pi^{2}\right)}, \\
D_{\Phi_{0} \Phi_{0}}= & \sigma^{2} \frac{3}{2} \frac{\pi^{2}}{A^{2} \Gamma\left(9+\varepsilon^{2} S^{2} \pi^{2}\right)}, \\
D_{A A}= & 18 \sigma^{2} \frac{A(2-A)}{\Gamma\left[9+\varepsilon^{2} S^{2}\left(\pi^{2}+12\right)\right]}, \\
D_{A \Gamma}= & 9 \sigma^{2} \frac{A-2}{9+\varepsilon^{2} S^{2}\left(\pi^{2}+12\right)}, \\
D_{\Gamma \Gamma}= & 3 \sigma^{2} \frac{\Gamma}{A} \frac{9(3-A)+\varepsilon^{2} S^{2}\left[\pi^{2}(3-A)+6 A\right]}{\left(9+\varepsilon^{2} S^{2} \pi^{2}\right)\left[9+\varepsilon^{2} S^{2}\left(\pi^{2}+12\right)\right]} .
\end{aligned}
$$

When solving the stochastic collective variable equations numerically, we will evaluate the components of the above matrix subject to the approximation

$$
\langle D(A, \Gamma)\rangle=D(\langle A\rangle,\langle\Gamma\rangle) .
$$

Expanding $D$ in a Taylor-series around the expectations of its arguments, we find:

$$
\begin{aligned}
D(A, \Gamma)= & D(\langle A\rangle,\langle\Gamma\rangle)+\frac{\partial}{\partial A} D(\langle A\rangle,\langle\Gamma\rangle)(A-\langle A\rangle) \\
& +\frac{\partial}{\partial \Gamma} D(\langle A\rangle,\langle\Gamma\rangle)(\Gamma-\langle\Gamma\rangle)+\ldots
\end{aligned}
$$

Taking into account that $\langle A-\langle A\rangle\rangle=0$ and the same for $\Gamma$, we see that the error in the equations of motion is of at least second order in the noise; thus the approximation (47) is consistent with the above approximations. The time dependence of $\langle A\rangle$ and $\langle\Gamma\rangle$ is assumed to be determined by the effects of damping alone. From (41-44) it is clear that this assumption involves approximations like (47) and hence, like (47), is justified for weak noise.

\section{Numerical results}

We have solved numerically the set of equations (41-44) and have obtained the results shown below by averaging over 10000 realizations. For comparison the spin dynamics of a chain of 1000 spins has been simulated. The spins have been subject to equation (3), which has been solved using the Heun algorithm [34], which provides an adequate treatment of the multiplicative couplings between 

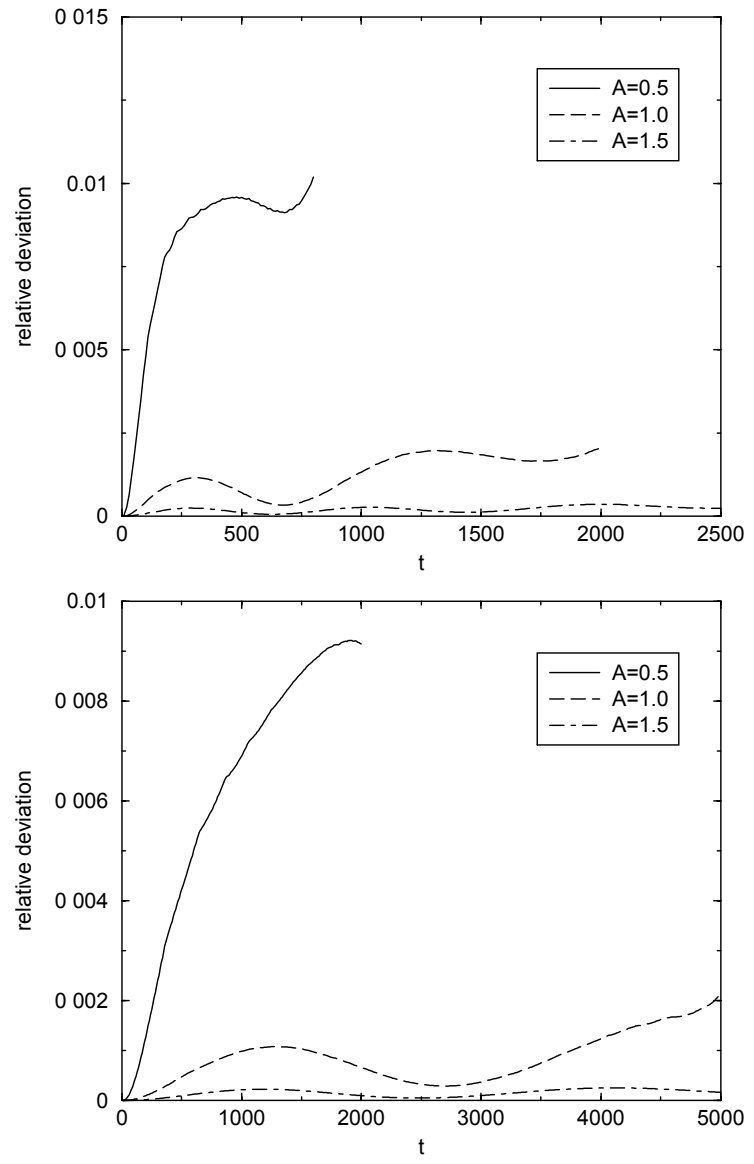

Fig. 1. Deviations of the simulated spin configuration from the ansatz with fitted collective variables. The damping parameter was $\varepsilon=0.01$. The initial values of $A$ are given in the insets. The initial values of $\Gamma$ were Top: $\Gamma=10$, Bottom: $\Gamma=20$. Some of the graphs had to be cut before $t=5000$, because the solitary wave reached the end of the chain before that time.

the components of the spins and of the noise, arising due to the vector product $\boldsymbol{S} \times \boldsymbol{b}$ in (3). The spin dynamics simulations have been extended up to 5000 time units and over 1000 realizations; we have made the choice $J=1$ and $S=1$. The collective variables $X, \Phi_{0}, A, \Gamma$ have been determined from spin dynamics by a fitting procedure that is described in detail in Appendix A.

First we check numerically how well the spin configuration is approximated by the ansatz. We take the values for the collective variables $X, \Phi_{0}, A$ and $\Gamma$ determined from the fit, plug them into the exact soliton solutions (8) and calculate the total squared deviation from the spin configuration obtained in a simulation with damping, but without noise. This quantity is then divided by $2 A \Gamma$, which gives the $z$-component of the total magnetization of an unperturbed soliton and which can thus be considered a measure for the "size" of the excitation. The quantity obtained in this fashion is shown in Figure 1 as a function of time. The results of Figure 1 have led us to restrict ourselves to values $A \geq 1$; our approach starts from an ansatz and we can only expect good results as long as the ansatz describes the spin configuration well. Figure 1 shows that this is the case for $A=1$ and $A=1.5$, whereas the relative deviations are much larger for $A=0.5$. Figure 2 compares results for the variance of the position $X$ of the solitary wave, obtained from spin dynamics simulations, numerical solution of the collective variable equations (41-44) and analytically (24). As can be seen from the left column, the analytical result (24) describes well the behaviour for short and intermediate times, whereas the numerical solution of (41-44) clearly deviates from the spin dynamics result. For long times, as shown in the right column, the situation is vice versa. This is due to the fact that the analytical prediction is in harmony with the way the position is measured in spin dynamics, whereas the collective variable equations obtained in the previous section are not (see Appendix B). For long times this is not relevant, as was surmised in [25] for the case of an explicit collective variable. The same argument given there applies to the implicit collective variables here. The noise produces fluctuations, amongst which are distortions of the soliton shape that cause a shift of the soliton position (translation modes). For a fixed distortion, different methods of determining the position from a simulated spin configuration can produce different results. However, these differences are limited in magnitude, as they are due to localized fluctuations. Therefore they become negligible when the standard deviation of the soliton position is comparable to the width of the excitation. Comparing the long time result for $A=1.5, \Gamma=20$ (Fig. 2, top) with the long time result for $A=1.5, \Gamma=40$ (Fig. 2, bottom) we see that for the case $\Gamma=40$ we have, at $t \approx 4500$, a standard deviation of about 5 , whereas for the case $\Gamma=20$ it is about 13 . Consistently, the relative deviations between spin dynamics and the solutions of (41-44) are much smaller in the case $\Gamma=20$. Naturally, the firstorder result of Section 2 does not hold for arbitrarily long times, as has been anticipated. The results of Figure 2 have been obtained at $k_{\mathrm{B}} T=0.001$. We get the same qualitative picture, the same agreement, for $k_{\mathrm{B}} T=0.002$ and $k_{\mathrm{B}} T=0.003$, but do not show the figures to save space. At $k_{\mathrm{B}} T=0.01$ we have observed that the code we are using runs into difficulties determining the parameters of the solitary wave, as thermal fluctuations distort the excitation strongly. Therefore we have restricted our investigations to the values of $k_{\mathrm{B}} T$ given above. In this context the reader should note that for $k_{\mathrm{B}} T \ll 1$ the mean thermal energy stored in one link of the chain in equilibrium is approximately $k_{\mathrm{B}} T$. If we consider $2 \Gamma$ as the number of spins "within" the solitary wave, i.e. in that region of the chain where the structure of the excitation clearly dominates, then for $\Gamma=20$ we have that on average a thermal energy of 0.04 is stored within the soliton at $k_{\mathrm{B}} T=0.001$. This is $20 \%$ of the soliton energy 0.2 , obtained as given after (8); at $T=0.003$ the thermal energy even amounts to $60 \%$ of the soliton energy. Hence the temperatures considered here are not small. Indeed, at $T=0.01$ the thermal energy is double the soliton energy and the distortions are so strong that the parameters of the solitary wave can no longer be determined. For the variable $\Phi_{0}$ we have obtained good agreement as well, see Figure 3 . The peaks occuring in the graph of the variance determined from spin 

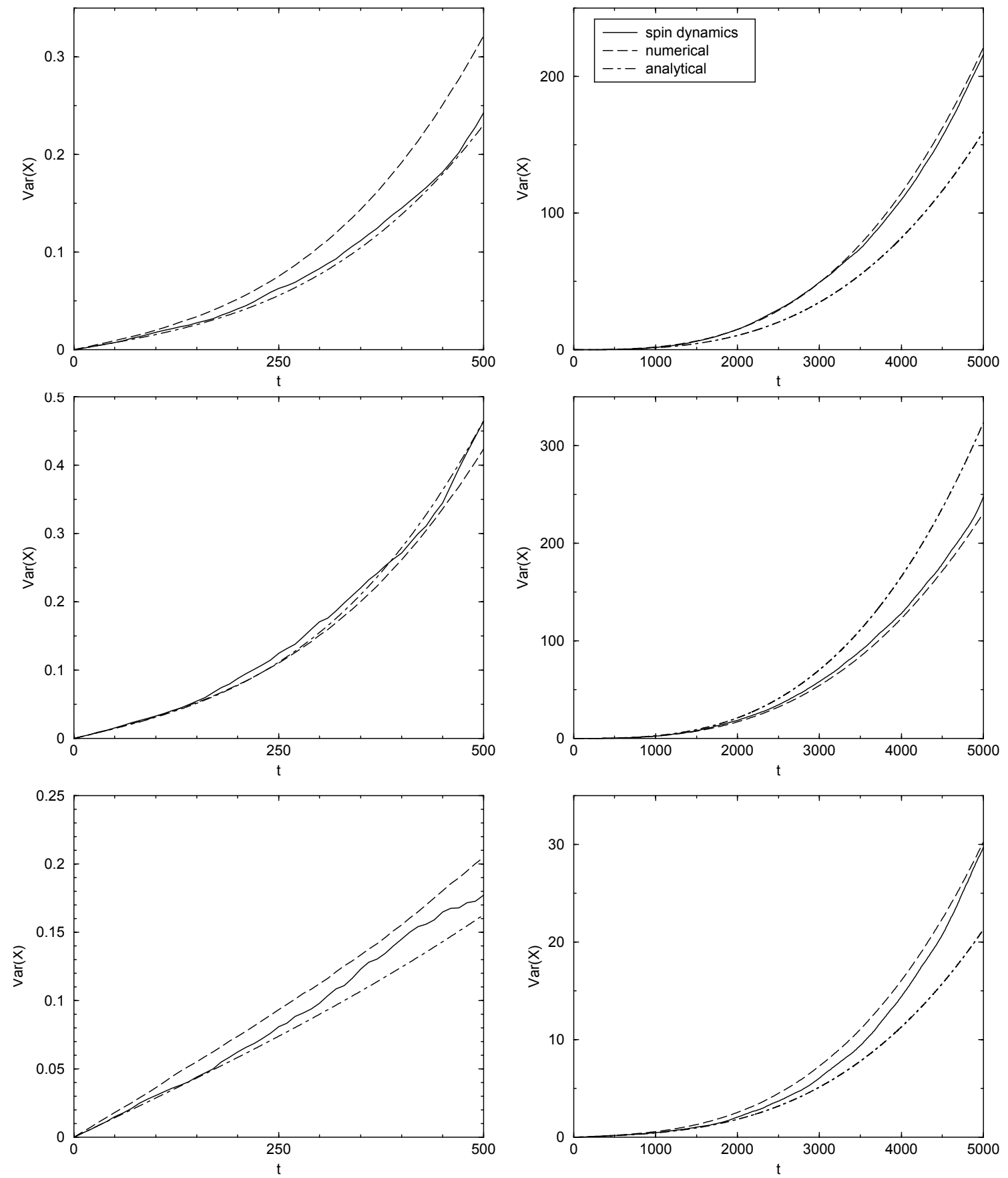

Fig. 2. The variance of the position as a function of time. The spin dynamics result (solid line) is an average over 1000 realizations, the result from the collective variable equations of Section 3 (dashed line) is an average over 10000 realizations. The dot-dashed line represents the analytical result (24). The plots on the left are zooms of the early stages of the plots on the right. For all cases the damping was $\varepsilon=0.01$ and the temperature $k_{\mathrm{B}} T=0.001$. Top: Initially $A=1.5, \Gamma=20$. Middle: Initially $A=1, \Gamma=20$. Bottom: Initially $A=1.5, \Gamma=40$.

dynamics are artifacts of the measuring procedure. The latter involves FORTRAN's ATAN2 function, which has a limited range, $(-\pi, \pi)$. The peaks occur when the mean value of $\Phi_{0}$ approaches a boundary of this range, so that for some realizations the value of $\Phi_{0}$ has already been shifted back to the opposite end of the range, whereas this back shifting has not yet occurred for other realizations.
Thus near the boundaries of the range, large differences of the order $2 \pi$ can occur between the values of $\Phi_{0}$ from different realizations. The "smoothing" of the jumps from $\pi$ to $-\pi$ in the spin dynamics graph for $\Phi_{0}$ has the same reason. On the other hand, when solving the collective variable equations, $\Phi_{0}$ is treated as a variable of unbounded range, the average over the realizations is calculated 

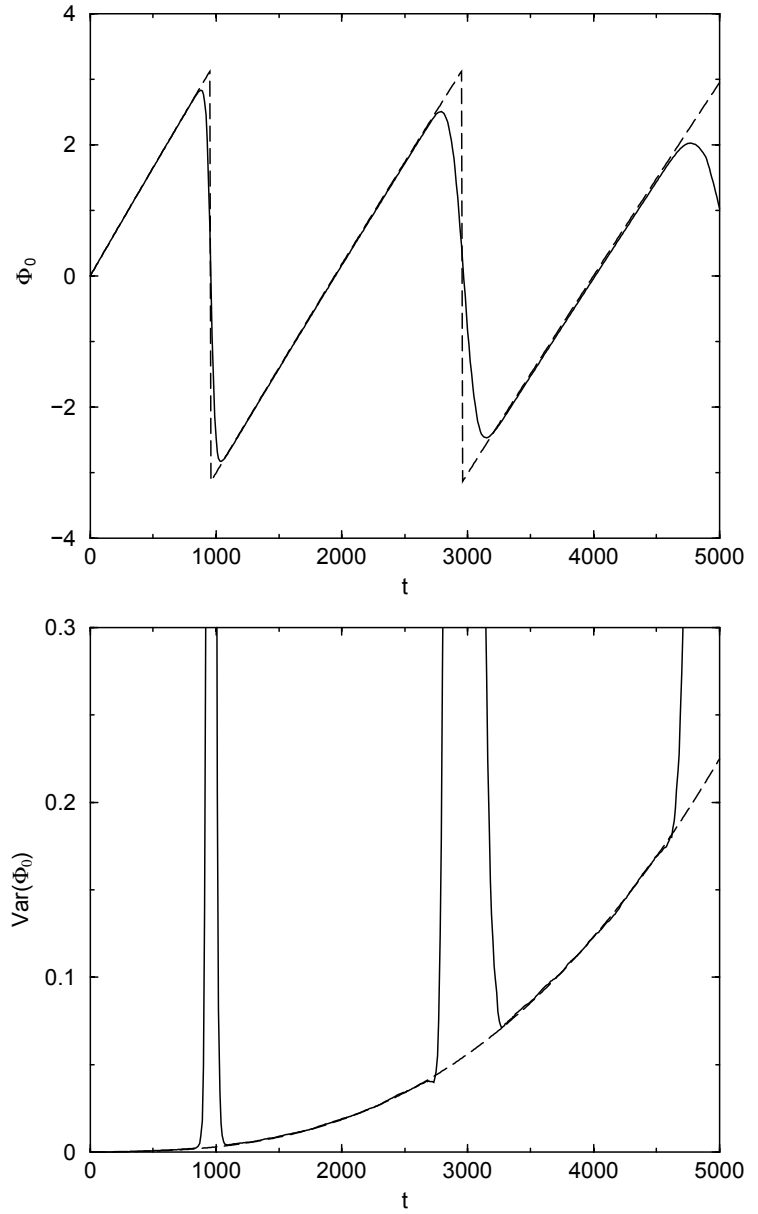

Fig. 3. Results for the collective variable $\Phi_{0}$. Initially: $A=$ $1.5, \Gamma=20$. The temperature was $k_{\mathrm{B}} T=0.001$, the damping $\varepsilon=0.01$. Shown are the mean value of $\Phi_{0}$ (top) and the variance of $\Phi_{0}$ (bottom). The solid lines give spin dynamics data (1000 realizations), the dashed line the results from numerically solving the collective variable equations of Section 3 (10000 realizations). The peaks in the variance have been cut off, because they are artifacts of the measuring procedure, as are the "smoothed" jumps from $\pi$ to $-\pi$ in the spin dynamics results for $\Phi_{0}$. See main text for further explanation.

and then this single value is reshuffled into the $(-\pi, \pi)$ range for better comparison with spin dynamics. Therefore we have sharp jumps for the dashed line.

In Figure 4 we show the mean values of $A$ and $\Gamma$. The tiny deviations of the spin dynamics result for $A$ from the solution of the collective variable equations are caused by the measuring procedure: the amplitude of these deviations increases, if the threshold used in the fit (see Appendix A) is increased. Apart from this, both graphs agree very well. For $\Gamma$ the spin dynamics result is slightly above the result of the collective variable equations. This difference increases with increasing temperature. The probable reason for this systematic deviation is a "dressing" of the soliton with fluctuations. Especially spike-like distortions appearing on the sides of the solitary wave can be suspected to increase the width determined from spin
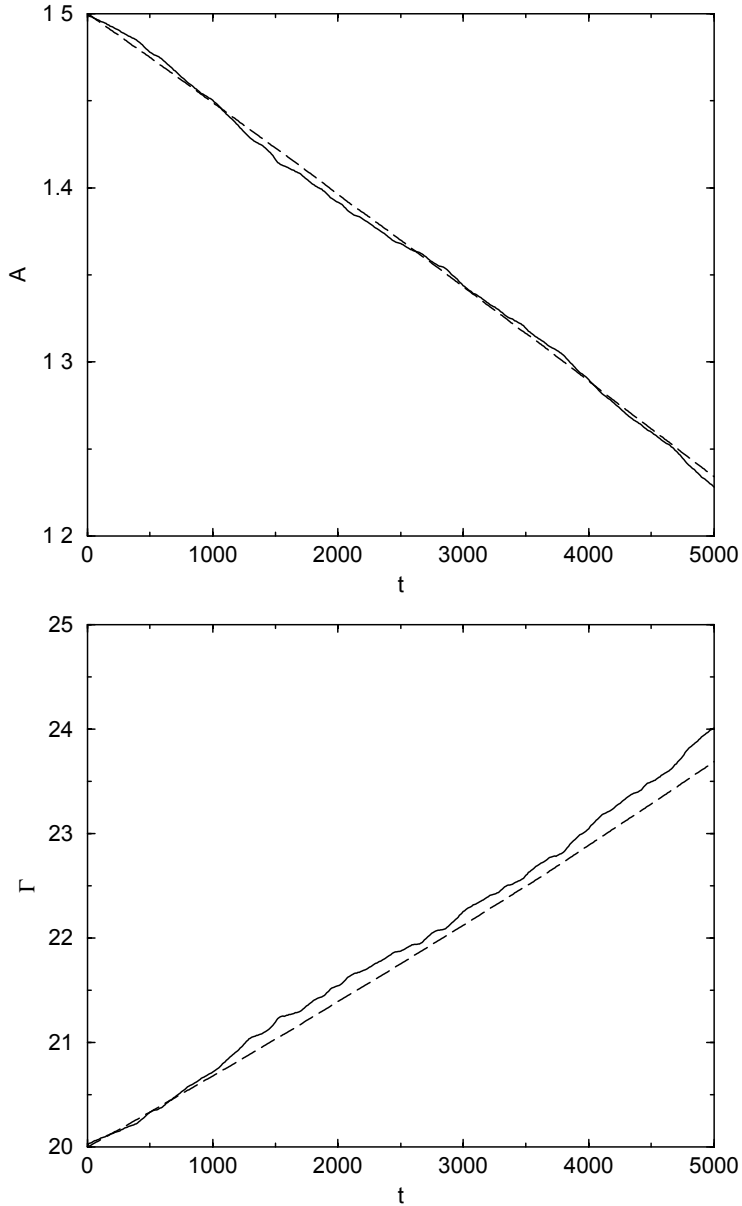

Fig. 4. Mean values for $A$ (top) and $\Gamma$ (bottom) at $k_{\mathrm{B}} T=$ 0.001 . The damping was $\varepsilon=0.01$. Shown are results from spin dynamics (solid line, 1000 realizations) and from the solution of the collective variable equations of Section 3 (dashed line, 10000 realizations).

dynamics. The fact that there is agreement here roughly during the first 1000 time units is quite consistent: At first, no fluctuations at all are present on the chain; after that time, as we have observed from spin dynamics, the system has approximately saturated energetically. But this also means that the fluctuations have fully developed then. The agreement for the variances of $A$ and $\Gamma$ is less satisfactory than for $X$ and $\Phi_{0}$, as can be seen from Figures 5 and 6 . This is due to the fact that the standard deviation of $A$ and $\Gamma$ is, even after 5000 time units, still small compared to the absolute values of $A$ and $\Gamma(<10 \%)$. Therefore it can be concluded that for these two quantities 5000 time units is still a short time. Thus deviations between spin dynamics and the numerical solution of the collective variable equations (41-44) are due to a disagreement between the way $A$ and $\Gamma$ are determined from spin dynamics and the way they enter the calculations. As the analytical calculations of Section 2 correspond to the fitting procedure employed in simulations (see Appendix B), the good agreement between the results of Section 2 and spin dynamics for short times supports this view. The mechanisms responsible for 

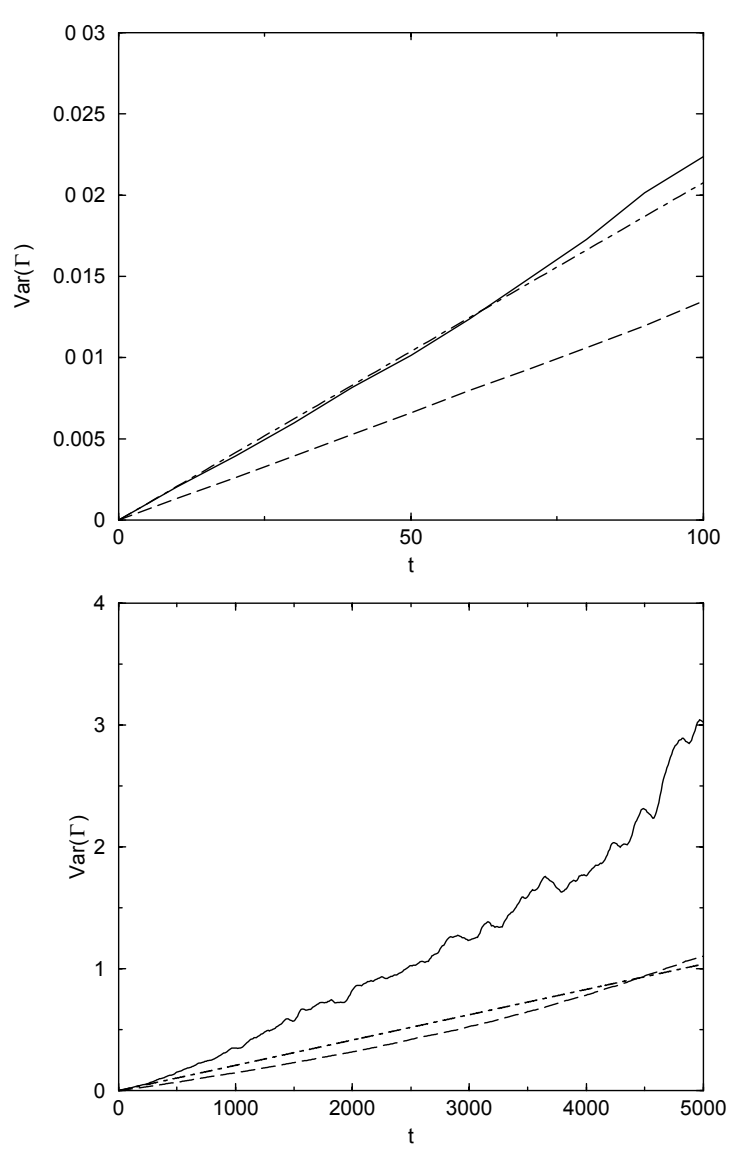

Fig. 5. Variance of $\Gamma$ at $k_{\mathrm{B}} T=0.001$ and $\varepsilon=0.01$. Initially the parameters were $A=1.5, \Gamma=20$. The solid line is the result of spin dynamics (1000 realizations), the dashed line has been obtained by solving the collective variable equations numerically (10000 realizations), and the dot-dashed line gives the analytical result (24). The top plot is a zoom of the early stages of the bottom one.

the discrepancies in the mean values of $A$ and $\Gamma$ also contribute to the deviations showing up in the variance.

\section{Conclusion}

On a classical, isotropic, ferromagnetic Heisenberg spin chain subject to Gilbert damping and Gaussian white noise coupled magnetically to the spins, solitary waves show diffusive motion, which has been investigated in this article by both analytical and numerical methods. Restricting ourselves to times short enough for damping to be neglected, we have derived analytical results for the time dependence of the variances of $X, A$ and $\Gamma$. Furthermore, we have obtained equations of motion for arbitrary collective variables, parametrizing the spin configuration of a general coherent excitation in a $d$-dimensional spin system, subject to Gilbert damping and magnetically coupled Gaussian white noise. The equations of motion are first-order in time and include a force composed of two parts: an intrinsic part, reflecting the interaction amongst
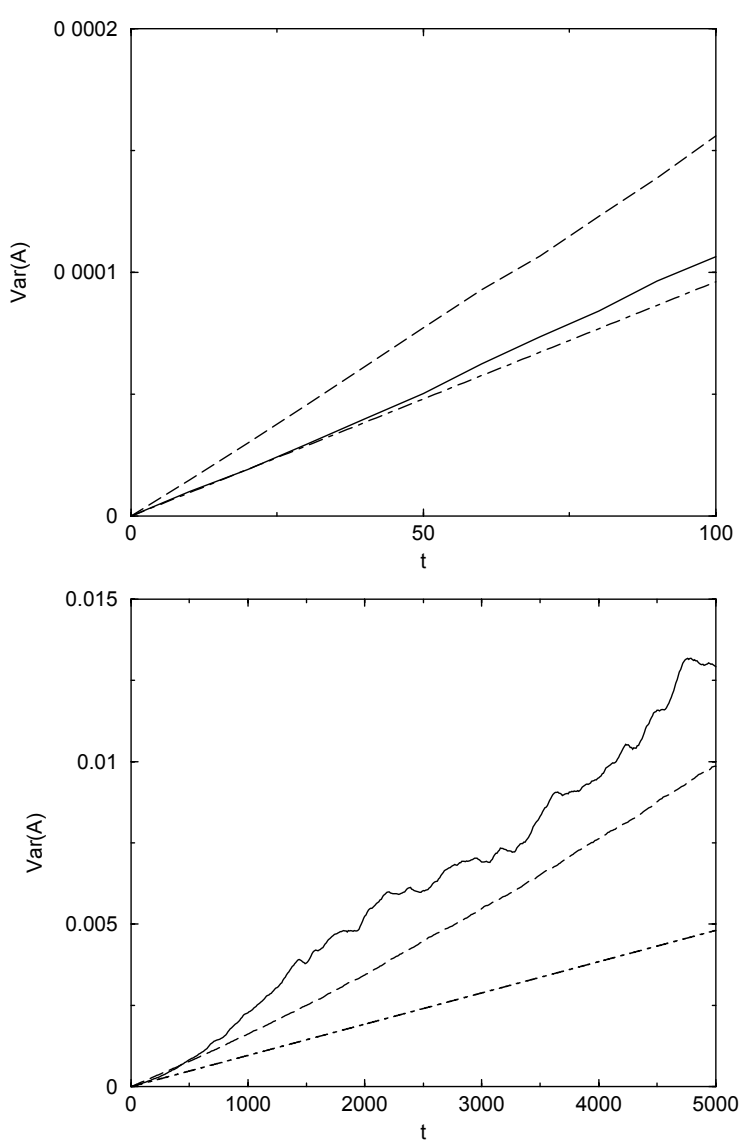

Fig. 6. As Figure 5, but for the collective variable $A$.

the spins on the collective level, and an external, additive white noise part. The latter derives from the noise coupled magnetically to the spins. The equations of motion have been specialized to the case of the Heisenberg chain, with collective variables $X, \Phi_{0}, A$ and $\Gamma$, and have been solved numerically. Both the analytical results and the numerical solutions of the equations of motion have been compared with data from spin dynamics simulations. For all four of the above collective variables we have observed that for short times there is a disagreement between data obtained from spin dynamics simulations and the numerical solutions of the equations of motion for the four collective variables given above. This is due to a lack of correspondence between the way these variables are measured in spin dynamics and the way they enter the calculations. As the method used to calculate the analytical results for the variances of $X, A$ and $\Gamma$ is more suited to the procedures employed in spin dynamics, we obtain agreement between spin dynamics and the analytical results for short times. There is no corresponding analytical result for $\Phi_{0}$, because, for reasons given in Section 2, this variable has not been involved in this approach. For long times, the results obtained by numerically solving the equations of motion in Section 3 match the spin dynamics data for $X$ and $\Phi_{0}$ very well. Within the time span of 5000 time units considered in this work, there is a systematic deviation between spin dynamics and numerical results from (41-44) 
for the variances of $A$ and $\Gamma$. This has been discussed in detail in Section 4, and hence we do not repeat this discussion here.

For the position $X$, which can be considered the most important of the four collective variables, because it possibly is the easiest to be accessed experimentally, we have found the following: There is a short time regime, in which the stochastic behaviour of the position is dominated by the direct effect of the noise on the position. This gives a linear dependence of the variance on time. The damping term, which is always taken to be weak, can safely be neglected. For intermediate times it is still possible to neglect a weak damping term, however, the stochastic fluctuations of $A$ and $\Gamma$ lead to stochastic fluctuations of the velocity (which is a function of $A$ and $\Gamma$ ) of the solitary wave. This in turn causes additional stochastic changes of the position. A term of cubic time dependence appears in the variance. In the long time regime damping can no longer be neglected. Together with the effects mentioned for short and intermediate times it affects the time dependence of the variance of $X$. The way the position is measured in spin dynamics is no longer important.

Fruitful discussions with A.R. Bishop (Los Alamos), Yu. Gaididei (Kiev), A. Kovalev (Kharkov) and G. Lythe (Madrid) are gratefully acknowledged. Work at Leganés was supported by DGESIC (Spain) through grant PB96-0119.

\section{Appendix A: Determining the collective variables from spin dynamics simulations}

Here we will explain in detail how the collective variables $X, \Phi_{0}, A$ and $\Gamma$ are determined from spin dynamics simulations. At first we discuss, for the sake of clarity, the hypothetical situation that there are no magnons in the system. Afterwards we turn to the real case.

If there are no magnons in the system, then in simulations we (hypothetically!) only have a solitary wave described by (8), where the collective variables $X, \Phi_{0}, A$ and $\Gamma$ depend stochastically on time. In particular, the $z$-component of the spin field depends on the collective variables as $S^{z}(r-X, A, \Gamma)$, with $X, A, \Gamma$ unknown. We define

$$
\begin{aligned}
& F_{X, A, \Gamma}(Y ; \tilde{A}, \tilde{\Gamma}):= \\
& \quad \int S^{z}(r-X, A, \Gamma) \frac{\partial S_{\mathrm{c}}^{z}}{\partial r}(r-Y, \tilde{A}, \tilde{\Gamma}) \mathrm{d} r .
\end{aligned}
$$

Here we use the index $c$ to distinguish the configuration of the system, $S^{z}(r-X, A, \Gamma)$, from the analytical solution (8), $S_{\mathrm{c}}^{z}(r-Y, \tilde{A}, \tilde{\Gamma})$. In the simulations $S^{z}$ will be replaced with the simulated spin configuration in (A.1); hence the distinction in notation. As long as we work under the hypothesis that there are no magnons, $S^{z}$ and $S_{\mathrm{c}}^{z}$ are the same functions, of course. Due to the symmetry of the soliton $(8), F_{X, A, \Gamma}(X, \tilde{A}, \tilde{\Gamma})=0$. A partial integration shows that $\frac{\mathrm{d} F_{X, A, \Gamma}}{\mathrm{d} Y}(X, \tilde{A}, \tilde{\Gamma})>0$. Both relations obtain independently of the values chosen for $\tilde{A}$ and $\tilde{\Gamma}$. It seems that a similar approach was followed in [3]. In our simulations we evaluate (a discretized version of) (A.1) for several trial values of $Y$, and fixed $\tilde{A}$ and $\tilde{\Gamma}$. The trial values for $Y$ are distributed symmetrically around the value of the position determined the previous time (for each realization and starting from a common, known initial position) in an interval of a total length equal to 40; the distance between two consecutive values is 0.25 (the lattice constant is unity). The values of $F_{X, A, \Gamma}$ that we have obtained in this way are then inspected for a change of sign from negative to positive as $Y$ is increased. The location of the zero on the $Y$-axis is estimated from a linear interpolation and considered as the position of the solitary wave.

In reality magnons are present in our simulations and unfortunately it turns out that magnons lead to a dependence of the position, determined as described above, and its variance, on the value of $\tilde{\Gamma}$. The position is still independent of $\tilde{A}$, because this quantity only appears as a positive, multiplicative factor of $F_{X, A, \Gamma}$ and thus changes neither the position of the zeros of this function on the $Y$-axis nor the sign of the slope of the function at such a zero. Because of the dependence on $\tilde{\Gamma}$, we modify the algorithm as follows:

Choosing a fixed value for $\tilde{A}$, e.g. $\tilde{A}=1$, we calculate a value $X_{i}$ for the position as described above, for each element of a set of 21 trial values $\Gamma_{i}$, which are distributed equidistantly in the range from $0.8 \Gamma_{0}$ to $1.8 \Gamma_{0}$, where $\Gamma_{0}$ is the initial width. As the width increases due to the damping, the range of trial values for $\Gamma$ has been chosen asymmetrically. Next, we calculate a value $A_{i}$ of the amplitude for each of these pairs $\left(X_{i}, \Gamma_{i}\right)$, yielding a collection of triplets $\left(X_{i}, A_{i}, \Gamma_{i}\right)$. The values $A_{i}$ are determined according to the formula

$$
\begin{aligned}
A_{i} & =\frac{\int\left(1-\frac{S^{z}}{S}\right) \operatorname{sech}\left(\frac{r-X_{i}}{\Gamma_{i}}\right)^{2} \mathrm{~d} r}{\int \operatorname{sech}\left(\frac{r-X_{i}}{\Gamma_{i}}\right)^{4} \mathrm{~d} r} \\
& =\frac{3}{4 \Gamma_{i}} \int\left(1-\frac{S^{z}}{S}\right) \operatorname{sech}\left(\frac{r-X_{i}}{\Gamma_{i}}\right)^{2} \mathrm{~d} r
\end{aligned}
$$

which can be derived in the following way: The quantity

$$
\int\left[S^{z}-S_{\mathrm{c}}^{z}\left(r-X_{i}, A, \Gamma_{i}\right)\right]^{2} \mathrm{~d} r
$$

obviously gives the squared deviation of the function $S_{\mathrm{c}}^{z}$ from an arbitrary spin configuration $S^{z}$. Considering $X_{i}$ and $\Gamma_{i}$ as fixed, differentiating (A.3) with respect to $A$, requiring that the result vanishes and solving for $A$ yields (A.2). So the $A_{i}$ represent the best values, as determined by a least square fit, for the amplitude, for given values of $X_{i}$ and $\Gamma_{i}$. In simulations, of course, the simulated spin configuration will be used for $S^{z}$ in (A.2).

As is obvious from the above, the quantities $X_{i}$ and $A_{i}$ depend on $\Gamma_{i}$. We therefore define, for a fixed simulated 
spin configuration,

$$
\Delta(\Gamma):=\int\left[S^{z}-S_{\mathrm{c}}^{z}(r-X(\Gamma), A(\Gamma), \Gamma)\right]^{2} \mathrm{~d} r,
$$

the squared deviation of the simulated spin configuration $S^{z}$ from the analytic solution $S_{\mathrm{c}}^{z}$, in which the parameters $X$ and $A$ are considered functions of $\Gamma$. The value of $\Gamma$ for which this function has a minimum should be considered the correct value of the width. In order to find the (approximate) location of this minimum on the $\Gamma$-axis, we evaluate from the simulated configuration, for each triplet $\left(X_{i}, A_{i}, \Gamma_{i}\right)$, the quantity $\Delta_{i}$ as a discretized version of $\Delta\left(\Gamma_{i}\right)$, where $X\left(\Gamma_{i}\right)=X_{i}, A\left(\Gamma_{i}\right)=A_{i}$. In calculating the $\Delta_{i}$ we only take into account sites where $S^{z}$ deviates more than a certain threshold from its groundstate value; otherwise the result would be dominated by thermal fluctuations in regions of the chain far from the solitary wave and therefore would no longer provide information about the parameters of the excitation. The threshold values have been $0.05,0.1$ and 0.2 for $k_{\mathrm{B}} T$ equal to $0.001,0.002$ and 0.003 , respectively. We choose the minimum value of the $\Delta_{i}$, and denote it $\Delta_{i_{M}}$. Next, we interpolate the three values $\Delta_{i_{M}}, \Delta_{i_{M}-1}$ and $\Delta_{i_{M}+1}$ with a parabola, which, like $\Delta$, has to be considered a function of $\Gamma$. The position of the minimum of the parabola on the $\Gamma$-axis is accepted as the definitive value of the width. With this value of the width we calculate again a value for the position and then for the amplitude, precisely as detailed above, and accept this triplet of numbers as values for position, amplitude and width of the solitary excitation in the simulated spin configuration.

What remains is to determine the angle $\Phi_{0}$. From (8) we see that the angle $\Phi$ is composed of the global part, $\Phi_{0}$, and a part that depends on $r$ and $X$. The latter will be denoted $\phi_{X}$ in the following. In simulations the total angle $\Phi$ can be measured easily for each of the spins of the chain, as $\Phi=$ ATAN2 $\left(S^{y}, S^{x}\right)$, ATAN2 denoting the FORTRAN function. Far from the soliton, $S^{y}$ and $S^{x}$ are practically zero. This would cause problems with the ATAN2 function. For this reason we restrict the calculation of the $\Phi$ values to the same spins as the calculation of the $\Delta_{i}$. We have determined $X, A$ and $\Gamma$, and hence can calculate $\phi_{X}$ and subtract it from $\Phi$. So we obtain a preliminary value for $\Phi_{0}$. As the range of the ATAN2 function is bounded to $(-\pi, \pi)$, whereas $\phi_{X}$, as given by (8), is unbounded, we carry out the subtraction in this way: We define $w x:=\cos \left(\Phi-\phi_{X}\right)=\cos (\Phi) \cos \left(\phi_{X}\right)+\sin (\Phi) \sin \left(\phi_{X}\right)$ and $w y:=\sin (\Phi) \cos \left(\phi_{X}\right)-\cos (\Phi) \sin \left(\phi_{X}\right)$ to obtain as a preliminary value $\Phi_{0}=\operatorname{ATAN2}(w y, w x)$. Due to local thermal fluctuations, this preliminary value will not be the same for all spins. Therefore the final value of the global angle $\Phi_{0}$ is determined by averaging over all preliminary values.

\section{Appendix B: Correspondence of calculations and simulations}

In this section we explain why the calculations of Section 2 correspond to the way the collective variables have been determined in simulations of the spin dynamics. Basically, the latter is a nonlinear least-square fit. We define, for purposes of this appendix, the function

$$
F(\boldsymbol{U}, \tilde{\boldsymbol{U}}):=\frac{1}{2} \int\left[S^{z}(\boldsymbol{U})-S_{\mathrm{c}}^{z}(\tilde{\boldsymbol{U}})\right]^{2} \mathrm{~d} r .
$$

Here $S^{z}$ represents (a continuum version of) the simulated spin configuration, depending on a set of time dependent collective variables $\boldsymbol{U}$. The function $S_{\text {c }}^{z}$ denotes the shapefunction which is to be fitted to the simulated spin configuration by varying the set of parameters $\tilde{\boldsymbol{U}}$. Note that there is a one-to-one correspondence between $\boldsymbol{U}$ and $\tilde{\boldsymbol{U}}$, each element of the latter being just a trial value for the corresponding element of the former. The necessary condition for the least-square fit of a given simulated spin configuration (i.e. with fixed $\boldsymbol{U}$ ) is a vanishing of the gradient of $F$ with respect to $\tilde{\boldsymbol{U}}$ :

$$
\frac{\partial F}{\partial \tilde{U}_{i}}=\int S^{z} \frac{\partial S_{\mathrm{c}}^{z}}{\partial \tilde{U}_{i}} \mathrm{~d} r-\int S_{c}^{z} \frac{\partial S_{\mathrm{c}}^{z}}{\partial \tilde{U}_{i}} \mathrm{~d} r=0,
$$

for each $\tilde{U}_{i}$. As the fit parameters $\tilde{\boldsymbol{U}}$ appear nonlinearly in the set of equations (B.2), solving these equations is not straightforward. Therefore we have instead used the procedure detailed in Appendix A, which can, however, still be considered a least-square fit. Taking the time derivative of the middle part of (B.2), we find

$$
\int \sum_{j} \frac{\partial S^{z}}{\partial U_{j}} \frac{\partial S_{c}^{z}}{\partial \tilde{U}_{i}} \mathrm{~d} r \dot{U}_{j}
$$

it should be noted that the $\tilde{U}_{i}$ are independent of time. Only the values obtained as solutions of (B.2) change with time as the spin configuration changes. Moreover, if we neglect all extended excitations and consider only the time dependence of $S^{z}$ caused by the time dependence of the collective variables $\boldsymbol{U}$, we can identify $S^{z}$ and $S_{\text {c }}^{z}$ and also $\partial S^{z} / \partial U_{i}$ with $\partial S_{\mathrm{c}}^{z} / \partial \tilde{U}_{i}$, because the dependence of $S^{z}$ and $S_{\text {c }}^{z}$ on $\boldsymbol{U}$ and $\tilde{\boldsymbol{U}}$, respectively, is identical. We can find out about the $\dot{U}_{j}$ in (B.3) as follows: We insert an ansatz like (26) in equation (9) and consider the $z$-component. This produces

$$
\sum_{j} \frac{\partial S^{z}}{\partial U_{j}} \dot{U}_{j}
$$

on the LHS, just as in (10) for the case of the soliton (8). Proceeding analogously to Section 2 and multiplying (B.4) with $\partial S^{z} / \partial U_{i}$, for each of the $U_{i}$, and integrating over $r$ gives a system of equations analogous to (11); again, the analogy is an identity for the soliton (8). Using the above identifications, we see that the LHS of this system is precisely the same as (B.3). Therefore the variables $U_{i}$ and in particular their time derivatives, appearing in this system of equations, are precisely those involved in the least-square fit. In this sense, the calculations of Section 2 correspond to the fit performed in spin dynamics, while the calculations of Section 3 do not. 


\section{References}

1. A.R. Bishop, J.A. Krumhansl, S.E. Trullinger, Physica D 1, 1 (1980).

2. A.M. Kosevich, B.A. Ivanov, A.S. Kovalev, Physics Reports 194, 117 (1990).

3. J. Dziarmaga, W. Zakrzewski, Phys. Lett. A 242, 227 (1998).

4. J. Dziarmaga, W. Zakrzewski, Phys. Lett. A 251, 193 (1999).

5. A.M. Kosevich, V.V. Gann, A.I. Zhukov, V.P. Voronov, J. Exp. Theor. Phys. 87, 401 (1998).

6. B.A. Ivanov, H.J. Schnitzer, F.G. Mertens, G.M. Wysin, Phys. Rev. B 58, 8464 (1998).

7. B.A. Ivanov, V.M. Murav'ev, D.D. Sheka, J. Exp. Theor. Phys. 89, 583 (1999).

8. P.G. Kevrekidis, M.I. Weinstein, Physica D 142, 113 (2000).

9. C. Rebbi, G. Soliani, Solitons and Particles (World Scientific, Singapore, 1984).

10. D.W. McLaughlin, A.C. Scott, Phys. Rev. A 18, 1652 (1978).

11. E. Mann, J. Math. Phys. 38, 3772 (1997).

12. E. Mann, J. Phys. A 30, 1227 (1997).

13. N.R. Quintero, A. Sánchez, F.G. Mertens, Phys. Rev. E 62, R60 (2000).

14. M.J. Rodriguez-Plaza, L. Vázquez, Phys. Rev. B 41, 11437 (1990).

15. N.R. Quintero, A. Sánchez, F.G. Mertens, Phys. Rev. Lett. 84, 871 (2000).

16. N.R. Quintero, A. Sánchez, F.G. Mertens, Phys. Rev. E 62, 5695 (2000)
17. Y. Wada, Prog. Theor. Phys. Supp. 113, 1 (1993).

18. N.R. Quintero, A. Sánchez, F.G. Mertens, Phys. Rev. E 60, 222 (1999).

19. N.R. Quintero, A. Sánchez, F.G. Mertens, Eur. Phys. J. B 16, 361 (2000).

20. F.G. Mertens, H.J. Schnitzer, A.R. Bishop, Phys. Rev. B 56, 2510 (1997).

21. A. Sánchez, A.R. Bishop, SIAM Rev. 40, 579 (1998).

22. R. Boesch, P. Stancioff, C.R. Willis, Phys. Rev. B 38, 6713 (1988).

23. H.J. Schnitzer, F.G. Mertens, A.R. Bishop, Physica D 141, 261 (2000).

24. R. Rajaraman, Solitons and Instantons (North Holland, Amsterdam, 1998).

25. M. Meister, F.G. Mertens, J. Phys. A 33, 2195 (2000).

26. T. Kamppeter, F.G. Mertens, E. Moro, A. Sánchez, A.R. Bishop, Phys. Rev. B 59, 11349 (1999).

27. C.W. Gardiner, Handbook of Stochastic Methods for Physics, Chemistry and the Natural Sciences (Springer, Berlin, 1983).

28. K. Nakamura, T. Sasada, Phys. Lett. A 48, 321 (1974).

29. M. Lakshmanan, T.W. Ruijgrok, C.J. Thompson, Physica A 84, 577 (1976).

30. J. Tjon, J. Wright, Phys. Rev. B 15, 3470 (1977).

31. H.C. Fogedby, J. Phys. A 13, 1467 (1980).

32. T. Kamppeter, F.G. Mertens, A. Sánchez, A.R. Bishop, F. Dominguez-Adame, N. Grønbech-Jensen, Eur. Phys. J. B 7, 607 (1999).

33. A.A. Thiele, Phys. Rev. Lett. 30, 230 (1973).

34. M. San Miguel, R. Toral, in Nonequilibrium Structures VI, edited by E. Tirapegui (Kluwer, Dordrecht, 1998). 